\title{
VALIDAÇÃO DE CONSTRUTOS PARA A PESQUISA COM DISTRIBUIDORES DE UMA CADEIA DE SUPRIMENTOS COM A TÉCNICA DE Q-SORT E ANÁLISE FATORIAL CONFIRMATÓRIA
}

\author{
CONSTRUCT VALIDATION FOR RESEARCH WITH DISTRIBUTORS OF A SUPPLY \\ CHAIN WITH Q-SORT TECHNIQUE AND CONFIRMATORY FACTOR ANALYSIS
}

\section{VALIDACIÓN DE CONSTRUCTOS PARA LA INVESTIGACIÓN CON DISTRIBUIDORES DE UNA CADENA DE SUMINISTRO CON LA TÉCNICA Q-SORT Y ANÁLISIS FACTORIAL CONFIRMATORIA}

\section{Emir José Redaelli}

Doutor em Administração pela Universidade do Vale do Rio dos Sinos (UNISINOS/RS)

emir.redaelli@terra.com.br

\section{Ely Laureano Paiva}

Doutor em Administração pela Universidade Federal do Rio Grande do Sul; Professor Adjunto da Fundação Getúlio Vargas

ely.paiva@fgv.br

\section{Rafael Teixeira}

Ph.D. em Management pela Clemson University, Carolina do Sul, EUA; Professor do Programa de Pós-graduação em Administração da UNISINOS/RS rafaelte@unisinos.br
Contextus

ISSNe 2178-9258

Organização: Comitê Científico Interinstitucional Editor Científico: Marcelle Colares Oliveira Avaliação : Double Blind Review pelo SEER/OJS Revisão: Gramatical, normativa e de formatação Recebido em 29/09/2013 Aceito em 27/04/2014 $2^{a}$ versão aceita em 02/06/2014 $3^{a}$ versão aceita em 20/06/2014

\section{RESUMO}

Os estudos sobre a gestão da cadeia de suprimentos se caracterizam pela relação entre fabricante e fornecedores, com poucos trabalhos sobre a relação entre fabricante e distribuidores. Este estudo tenta preencher essa lacuna na literatura ao propor a validação de construtos teóricos que contribuam para futuras pesquisas sobre as relações existentes na integração a jusante na cadeia de suprimentos. Mais especificamente, este estudo propõe a construção e a validação dos seguintes construtos: monitoramento do desempenho dos distribuidores, envolvimento direto entre fabricante $\mathrm{e}$ distribuidores, recursos e transferência de conhecimentos, e desempenho geral e operacional dos distribuidores. Para atingir esse objetivo, uma série de etapas foi desenvolvida para que a construção de escalas válidas e confiáveis fosse alcançada. Foi utilizada a técnica questionnaire sorting com doze doutores na área bem como uma análise fatorial confirmatória com dados de 199 empresas participantes da pesquisa. Os resultados levaram ao refinamento e a validação do instrumento. Palavras-chave: Gestão da cadeia de suprimentos. Fabricantes. Distribuidores. Integração.

\begin{abstract}
Studies about supply china management are characterized by a great emphasis on the relationship between manufacturer and its supplies and few studies have focused on the relationship between manufacturer and its distributors. This study attempts to fulfill this gap in the literature by proposing the validation of theoretical constructs that contribute for future studies about the existent relationships in the downstream supply chain. More specifically, this study aims to construct and validate the following constructs: monitoring of distributor performance, direct involvement between manufacturer and distributor, resources and knowledge transfer, overall and operational performance of distributors. To do this, many steps were conducted to develop reliable and valid constructs. The questionnaire sorting technique as employed with twelve professors holding a Ph.D. as well as a confirmatory factor analysis was conducted with results obtained from 199 firms participating in the survey. The results led to improvement and validation of the measurement instrument.
\end{abstract}

Keywords: Supply chain management. Manufacturer. Distributor. Integration. 


\section{RESUMEN}

Los estudios sobre la gestión de la cadena de suministro se caracteriza por la relación entre el fabricante y el distribuidor, con pocos estudios sobre la relación entre el fabricante y sus distribuidores . Este estudio intenta llenar este vacío en la literatura , proponiendo la validación de constructos teóricos que contribuyen a los estudios futuros de las relaciones en la integración aguas abajo en la cadena de suministro. Más en concreto , este estudio se propone la construcción y validación de las siguientes construcciones : el seguimiento del desempeño de los distribuidores, el compromiso directo entre el fabricante y los distribuidores, los recursos y la transferencia de conocimientos y el rendimiento operativo global de distribuidores. Para lograr este objetivo, una serie de medidas se han desarrollado para se consigue la construcción de escalas válidas y fiables . La técnica de la ordenación cuestionario consta de doce médicos de la zona, así como un análisis factorial confirmatorio con datos de 199 empresas que participan en la encuesta. Los resultados llevaron al refinamiento y validación del instrumento.

Palabras-clave: Gestión de la cadena de suministro. Fabricantes. Distribuidores. Integración.

\section{INTRODUÇÃO}

A literatura sobre a gestão da cadeia de suprimentos é caracterizada por estudos sobre a integração dessa cadeia com um direcionamento a montante, ou seja, entre fabricantes e fornecedores (WAGNER; KRAUSE, 2009; MODI; MABERT, 2007; PRAHINSKI; FAN, 2007; WAGNER, 2006; PRAHINSKI; BENTON， 2004; HUMPHREYS; LI; CHAN, 2004; KRAUSE; SCANNELL; CALANTONE, 2000; PURDY; ASTAD; SAFAYENI, 1994). A cadeia de suprimentos, por outro lado, envolve não somente a relação entre fabricante e fornecedores, mas também a relação entre fabricante e distribuidores. Entretanto, existem poucos estudos na literatura versando sobre a interação entre fabricante e distribuidores.

O marketing industrial tem abordado o tema da relação entre fabricante e distribuidores, enfatizando aspectos da relação de poder e governança dos canais. Zhou e Benton Jr. (2007) trataram da troca de informações nas práticas da gestão da cadeia de suprimentos. Wren (2007) analisou a relação da integração vertical com estratégia, poder e controle. Bellin (2006) abordou a efetividade do sistema de canais de distribuição. Mehta, Dubinsky e Anderson (2002) analisaram a formulação das estratégias dos canais, desde o seu arranjo e a seleção dos parceiros, a motivação e coordenação para o alinhamento das estratégias, entre outras etapas. Dyer e Singh (1998) avaliaram a formação de parcerias do chamado capital relacional entre as empresas da cadeia de suprimentos, enquanto Dyer e Nobeoka (2000) conduziram um estudo sobre o relacionamento entre fabricante e fornecedores que é sustentado pelo compartilhamento dos conhecimentos. Entretanto, ainda existe espaço para uma contribuição referente à integração entre 
fabricante e distribuidores e a relação com o desempenho da cadeia de suprimentos, buscando responder a seguinte questão: como os objetivos dos fabricantes podem ser transformados em programas de desenvolvimento e monitoramento dos distribuidores, alinhando os interesses de todos ao longo da cadeia de suprimentos?

Uma segunda lacuna na literatura refere-se à questões metodológicas, principalmente de ordem métrica, no que tange à falta de instrumentos que permitam a medição de fenômenos organizacionais relativos à cadeia de suprimentos. Muitos estudos têm sido desenvolvidos de forma a prover instrumentos que capturem parte dessa realidade organizacional na cadeia de suprimentos (HARTLEY; CHOI, 1996; KRAUSE et al., 2000). Entretanto, são poucos os instrumentos para a coleta de dados sobre os problemas existentes a jusante na cadeia.

Os elementos-chave para uma compreensão da relação entre fabricante e distribuidores envolvem: (i) atividades formais e informais, tomadas pelo fabricante ou pela organização que detém a governança da estrutura de distribuição e (ii) ferramentas desenvolvidas para os distribuidores utilizadas para aumentar a capacidade dos envolvidos com foco nas metas do fabricante. Esses elementos são encontrados nos estudos sobre a relação entre fabricante e fornecedores
(HANDFIELD et al., 2000; KRAUSE, 1997, 1999; KRAUSE; HANDFIELD; SCANNELL, 1998; KRAUSE et al, 2000; PURDY et al., 1994; WATTS; HAHN, 1993) e serão utilizados aqui para entender a relação entre fabricante e distribuidores.

Portanto, na tentativa de contribuir para o entendimento dos problemas relacionados a jusante na gestão da cadeia de suprimentos, este artigo tem o objetivo de apresentar e validar um conjunto de construtos considerados importantes para o entendimento da relação entre fabricantes de produtos e distribuidores. Mais especificamente, o trabalho tem o objetivo de construir e validar os seguintes construtos: monitoramento do desempenho do distribuidor pelo fabricante, envolvimento direto do fabricante na relação com o distribuidor e os recursos e conhecimentos transferidos do fabricante para o distribuidor.

Para isso, foi realizado um processo de construção das escalas para cada construto, em que a técnica questionnaire sorting (Q-sort) e a análise fatorial confirmatória são utilizadas para a validação de tais escalas. A principal contribuição deste trabalho é a construção e a validação de um instrumento para a medição dos construtos supracitados, de modo a permitir que outros estudos sobre a cadeia de suprimentos possam evoluir a 
partir dos construtos discutidos no trabalho.

\section{REVISÃO DA LITERATURA}

\subsection{Cadeia de Suprimentos}

A cadeia de suprimentos é definida como um conjunto de três ou mais organizações diretamente envolvidas com o fluxo de produtos a montante e a jusante, incluindo também os serviços, os aspectos financeiros e as informações de uma fonte até os consumidores (MENTZER et al., 2001). Como os canais de distribuição e vendas passam a ser fatores relevantes para os resultados empresariais, o seu gerenciamento efetivo se torna importante principalmente em mercados dinâmicos e altamente competitivos. Pelo grande impacto na competitividade, os canais de distribuição e vendas passam a ser a extensão das fábricas nos mercados em que atuam (PORTER, 1999).

A seguir, será apresentado cada um dos construtos discutidos na introdução, para os quais serão construídas as escalas de medição.

\subsection{Desenvolvimento na Cadeia de Suprimentos}

As empresas compradoras têm enfatizado o desenvolvimento de relacionamentos colaborativos e cooperativos de longo prazo com seus principais fornecedores (WILSON, 1995). Essa é a base dos fundamentos do presente estudo, em que são avaliadas as iniciativas do fabricante para contribuir para o desenvolvimento dos seus distribuidores. O monitoramento dos resultados de desempenho e o envolvimento direto são as bases da integração para a transferência de conhecimentos que propiciam o incremento nos resultados dos canais. As seções a seguir descrevem os elementos mais usuais encontrados na literatura relacionados ao desenvolvimento da cadeia de suprimentos.

\subsubsection{Monitoramento do desempenho}

O monitoramento do desempenho pode ser utilizado para o acompanhamento dos resultados dos distribuidores, tendo como base a capacidade de atendimento às demandas dos clientes e os elementos de coordenação (FROHLICH; WESTBROOK, 2001). Assim, para que os objetivos sejam de fato perseguidos pelos distribuidores de um fabricante é necessário o monitoramento do desempenho desses distribuidores. Quando um distribuidor conhece as metas estabelecidas por um fabricante, ele pode melhor colocar seus recursos para atingi-las (FRAZIER, 1999). O processo de retorno dos resultados, ou feedback, pode então ser considerado como uma atividade central no monitoramento, 
que requer troca de informações entre os canais e o fabricante e vice-versa. Custos associados ao monitoramento são partes dos custos da governança da rede e podem ser tratados como custos de transação. Os investimentos específicos em ativos de relacionamento em ambos os parceiros da díade fabricante e distribuidor, ou em investimentos em ativos específicos, são idiossincráticos para aquele relacionamento e têm pouco valor para os relacionamentos fora da cadeia de valor (WILLIAMSON, 1985).

Com isso, os investimentos feitos pelo fabricante no desenvolvimento dos distribuidores podem ser tomados como idiossincráticos, sendo capazes de reduzir os níveis de oportunismo (do fabricante em relação aos canais) e de servir como sinais intencionais de continuidade de relacionamentos comerciais com os canais (HEIDE; JOHN, 1992). Diversos estudos demonstram que o alinhamento na cadeia de suprimentos a montante e a jusante, com o incremento das capacidades e a qualificação de mais parceiros, é benéfico para o desempenho da cadeia. Nesse sentido, a troca de conhecimentos e a integração são fatores críticos para o desempenho do negócio (TEECE, 2007). Essa troca é estabelecida através dos mecanismos de comunicação existentes ao longo da cadeia de suprimentos, incluindo assim os canais de distribuição e vendas.

\subsubsection{Envolvimento direto}

O envio de pessoal da fábrica para as unidades dos distribuidores pode ser visto como uma forma de envolvimento direto entre as partes (HARTLEY; CHOI, 1996; KRAUSE et al., 2000). A prática da transferência de funcionários entre empresas é comum e usual nas organizações japonesas, definida por shukko, com vista a auxiliar os fornecedores das grandes empresas a manterem controle sobre os processos regulares de fornecimento. Essa prática é responsável pela criação de uma identidade de rede e serve como mecanismo para a transferência de conhecimentos do fabricante para os fornecedores (DYER; NOBEOKA, 2000).

Na Toyota, ao longo da década de 1990, foram transferidos mais de 120 empregados por ano para outras firmas, conforme relataram Dyer e Nobeoka (2000). Alguns funcionários foram transferidos de forma permanente, como diretores das unidades de fornecimento, enquanto outros, de forma temporária, como auxiliares, para tornar as operações das conveniadas mais eficientes. Segundo o estudo de Dyer e Nobeoka (2000), as transferências relatadas em ambos os casos se deram porque os fornecedores necessitavam desenvolver capacidades e conhecimentos específicos que a força de 
trabalho não possuía. Dessa forma, os conhecimentos técnicos são transferidos, da mesma maneira que a filosofia de trabalho, a tecnologia e o know-how do fabricante são compartilhados com os fornecedores. Com essa prática, os autores explicam que é buscada uma identidade comum para a cadeia de suprimentos como um todo.

A prática junto aos parceiros na Toyota ocorre através de reuniões mensais que facilitam a troca de conhecimentos. Esses encontros facilitam 0 desenvolvimento da comunicação entre os agentes, podendo ser complementados por visitas para disseminar as melhores práticas entre diversas unidades de produção (DYER; NOBEOKA, 2000). Para Dyer e Nobeoka (2000), o envolvimento direto via interações face a face favorece o processo de transferência de conhecimentos e cria uma maior interação social entre os envolvidos.

\subsubsection{Recursos e Transferência de Conhecimentos}

Para Kogut e Zander (1992), a firma é uma comunidade social especializada na velocidade e na eficiência de criação e de transferência de conhecimentos. Esse conhecimento pode ser compreendido como o know-how e a informação disponível na firma. O conhecimento social que está imbricado nas competências dos indivíduos e nos princípios do trabalho organizado é que se constitui nas bases do know-how disponível. Conhecimento, como informação, implica saber o que algo significa, enquanto a descrição do como usar esse conhecimento significa saber fazer uma determinada ação de forma eficiente.

Os tipos de conhecimento podem ser transferidos de várias formas. O tipo explícito pode ser repassado pelos manuais, pelas guias, pelo relatório, entre outros veículos, enquanto o conhecimento tácito, pelas rotinas organizacionais (TEECE; PISANO; SHUEN, 1997) e pelas atividades distintas desenvolvidas pelos funcionários na execução dos processos empresariais. Esses empregados podem ser separados das atividades, e essa é uma qualidade que somente se torna evidente na atividade (SPENDER, 1996). A aprendizagem, portanto, pode requerer interações frequentes entre grupos pequenos, geralmente obtida pelo desenvolvimento de uma linguagem única ou de códigos com significado compartilhados (KOGUT; ZANDER, 1992).

As rotinas reforçam a ideia de dependência de trajetória da firma (AUGIER; TEECE, 2009) porque a firma pode ser vista como uma coleção de 
rotinas impregnadas de conhecimentos tácitos que podem ser melhorados com novos conhecimentos adquiridos via aprendizagem. As rotinas podem ser codificadas e formalizadas através de instruções de trabalho ou impregnadas nos membros da organização. A capacidade de codificar o conhecimento se refere à habilidade da firma de estruturar o conhecimento num conjunto de regras identificadas e de relacionamentos facilmente comunicados (KOGUT; ZANDER, 1992).

Wahab, Rose e Osman (2011) reportaram que, para os parceiros de uma rede de empresas, a capacidade de transferência de conhecimentos tem efeitos significativos no repasse de conhecimentos tácitos e explícitos, sendo o efeito sobre o grau de conhecimento explícito ligeiramente mais forte do que o resultado sobre o grau de conhecimento tácito. No estudo desses autores, o repasse de conhecimentos formais era mais significativo do que a troca pessoal de conhecimentos, o que exige mais esforços na sistematização do conhecimento formalizado.

A palavra transferência é usada como difusão, para enfatizar o movimento do conhecimento retido pela organização (SZULANSKI, 1996). Nesse sentido, a transferência das melhores práticas pode ser entendida como a replicação das rotinas organizacionais (WINTER, 1995). Contatos informais entre grupos de pessoas de diferentes unidades de negócios de uma cadeia podem ser estabelecidos para o compartilhamento de um conhecimento que está disponível numa outra unidade daquela cadeia. Os mecanismos de integração social ativam o reconhecimento de valor no conhecimento, e a vantagem competitiva decorrente é o aumento da flexibilidade, do poder de inovação e do desempenho superior (TODOROVA; DURISIN, 2007).

Neste estudo, a transferência de conhecimentos será analisada sob a perspectiva de capabilities, reforçadas através de treinamentos técnicos e operacionais e de educação corporativa, conforme Krause et al. (2000), Monczka, Trent e Callahan (1993), Galt e Dale (1991) e Humphreys et al. (2004). Dessa forma, serão considerados os seguintes aspectos: (i) as contribuições dos treinamentos do fabricante para o aumento de conhecimentos técnicos sobre os produtos; (ii) a contribuição dos treinamentos do fabricante para o aumento de conhecimentos sobre os clientes; (iii) a contribuição dos treinamentos do fabricante para o aumento de conhecimentos do distribuidor sobre o mercado; (iv) a contribuição dos treinamentos do fabricante para o aumento de conhecimentos sobre os serviços de 
assistência técnica prestados pelo distribuidor.

\subsection{Desempenho Operacional e Geral}

Assume-se que o desempenho operacional do distribuidor é central para o desempenho do fabricante, assim como o sucesso do fabricante é decorrente do desempenho operacional de seus fornecedores $\quad($ SHIN; $\quad$ COLLIER; WILSON, 2000).

Prahinski e Benton

esclarecem que as medidas de desempenho podem ser tanto financeiras quanto operacionais (não-financeiras). Medidas operacionais de desempenho podem ser classificadas em duas grandes linhas: (i) pelos fatores-chave para a competitividade, tais como qualidade, prazo de entrega, preço, serviços e flexibilidade; (ii) fatores internos de operações, como defeitos, programação de operações e de entregas, controle de custos, entre outros.

Como uma cadeia de suprimentos é um sistema que abrange diversas empresas que atuam conjuntamente, os indicadores devem ser uma síntese coletiva dos resultados, ao mesmo tempo que isolam e identificam as contribuições individuais. Isso pode envolver medidas de operações e de utilização dos ativos (BOWERSOX; CLOSS; COOPER, 2006).
Um fabricante, ao estabelecer metas e institucionalizar a avaliação do desempenho dos distribuidores, pode contribuir para o desenvolvimento das capabilities ao longo de toda uma cadeia. Essa sistemática possibilita a melhoria do desempenho global e essa melhoria pode suportar, além dos objetivos presentes, os objetivos futuros. Prahinski e Benton (2004) propuseram o que denominaram Programa de Desenvolvimento de Fornecedores (SDP, sigla em inglês para Supplier Development Program), baseado em estratégias de comunicação.

Desse modo, considera-se que monitorar e avaliar o desempenho também envolve o ato de desenvolver instrumentos de retroalimentação (feedback). Neste estudo, o desempenho operacional será analisado sob a perspectiva dos processos operacionais, conforme Arbache (2004), Wagner (2006) e Krause et al. (2000). Para tanto, serão investigadas as melhorias nos processos operacionais a partir da percepção de: (i) reclamações de clientes com o canal; (ii) custos operacionais do canal; (iii) prazos de entrega dos serviços disponibilizados pelo canal; (iv) melhorias em todos os processos empresariais do canal.

Por outro lado, o desempenho geral do distribuidor será analisado aqui sob a perspectiva dos resultados da unidade de negócio e do desempenho geral do canal 
de distribuição e vendas, conforme Arbache (2004), Jaworski e Kohli (1993). Para isso, serão investigados: (i) o retorno obtido sobre o capital investido; (ii) a participação de mercado; (iii) a lucratividade; (iv) o aumento de vendas.
Após essa discussão da literatura, é possível apresentar um modelo de análise que esboce a relação entre os elementos previamente discutidos e que sirva de guia para a análise posterior. A figura 1 resume o modelo de análise utilizado.

\subsection{Modelo de Análise}

Figura 1 - Modelo de análise

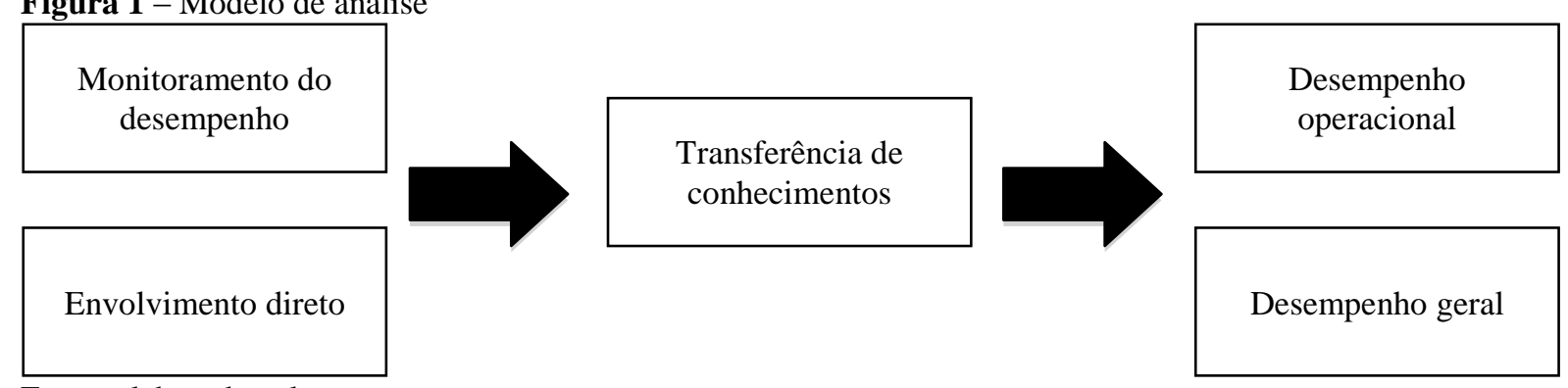

Fonte: elaborada pelos autores

O modelo de análise apresentado na figura 1 mostra a relação entre os conceitos abordados na revisão da literatura. De forma resumida, o modelo sugere que o monitoramento do desempenho do distribuidor no canal de vendas e o envolvimento direto do fabricante com esse distribuidor proporciona a troca de conhecimentos entre esses atores da cadeia de suprimentos. O monitoramento do desempenho é uma atividade que permite a comunicação mais formal e frequente entre o distribuidor e o fabricante, de forma que o distribuidor receba informações sobre como está a sua atuação no canal de vendas. $\mathrm{O}$ distribuidor pode ficar mais atento aos objetivos, às ações e aos recursos direcionados para o atendimento das demandas dos clientes (FRAZIER, 1999). De forma semelhante, é o efeito do envolvimento direto do fabricante com os funcionários e atividades desenvolvidas pelo distribuidor.

O envolvimento direto dos funcionários do fabricante com os funcionários e as atividades do fornecedor ou, no caso deste artigo, com os do distribuidor, já mostrou resultados que levam à conclusão de que tal prática gera a transferência de conhecimentos (DYER; NOBEOKA, 2000; HARTLEY; CHOI, 1996). Isso acontece porque tal envolvimento permite que o conhecimento tácito dos funcionários do fabricante seja repassado aos funcionários do distribuidor através de atividades realizadas em 
conjunto, reuniões de acompanhamento das atividades ou até mesmo pelas conversas informais. Assim, seja através de mecanismos mais formais, como o monitoramento do desempenho, seja através de mecanismos mais informais, como o envolvimento direto, ambas as atividades permitem que algum conhecimento técnico seja transmitido do fabricante para o distribuidor.

A transferência de conhecimentos também permite que o distribuidor adquira mais conhecimentos (ARGOTE; INGRAM, 2000) e possa, portanto, desempenhar suas atividades de forma mais adequada e alinhada com as práticas desenvolvidas pelo fabricante. Por ter mais conhecimento dos seus próprios produtos ou por ter adquirido mais informação sobre o processo de gerenciamento de outros distribuidores, o fabricante, portanto, pode repassar mais facilmente seus próprios produtos e serviços ao distribuidor com o objetivo de melhorar, de forma geral, o desempenho dele mesmo e as práticas do fabricante e da cadeia de suprimentos como um todo.

Essa transferência de conhecimentos permite que o distribuidor saiba a melhor forma de proceder para resolver um determinado problema ou executar melhor uma atividade (HUNTER; BEAUMONT; SINCLAIR, 1996; LANGFIELD-SMITH; GREENWOOD,
1998; WAGNER, 2006). Os funcionários podem aprender as melhores práticas sem ter que passar pelos mesmos erros pelos quais outros distribuidores já passaram e que foram incorporados pelo fabricante. Dessa forma, a transferência de conhecimentos tem implicação no desempenho operacional e geral do distribuidor.

\section{DELINEAMENTO METODOLÓGICO}

\subsection{Construção do instrumento}

A estratégia metodológica envolveu uma série de etapas que podem ser visualizadas na figura 2. Cada uma dessas etapas será descrita ao longo desta seção, porém em subseções pertinentes a cada etapa.

No processo de construção do instrumento de pesquisa, foi feita, primeiramente, uma revisão da literatura para identificar e definir o domínio dos construtos utilizados na pesquisa. Os construtos investigados foram: (i) monitoramento do desempenho; (ii) envolvimento direto; (iii) transferência de conhecimentos; (iv) desempenho operacional; (iv) desempenho do canal de distribuição e vendas. Em seguida, várias escalas foram identificadas na literatura para servir de base para essa construção e para posterior uso do instrumento de pesquisa. Quando as escalas identificadas 
não satisfaziam o domínio dos construtos,

eram criadas.

elas eram modificadas ou novas escalas

Figura 2 - Processo de desenvolvimento do instrumento de pesquisa survey omitindo os caminhos de feedback do modelo

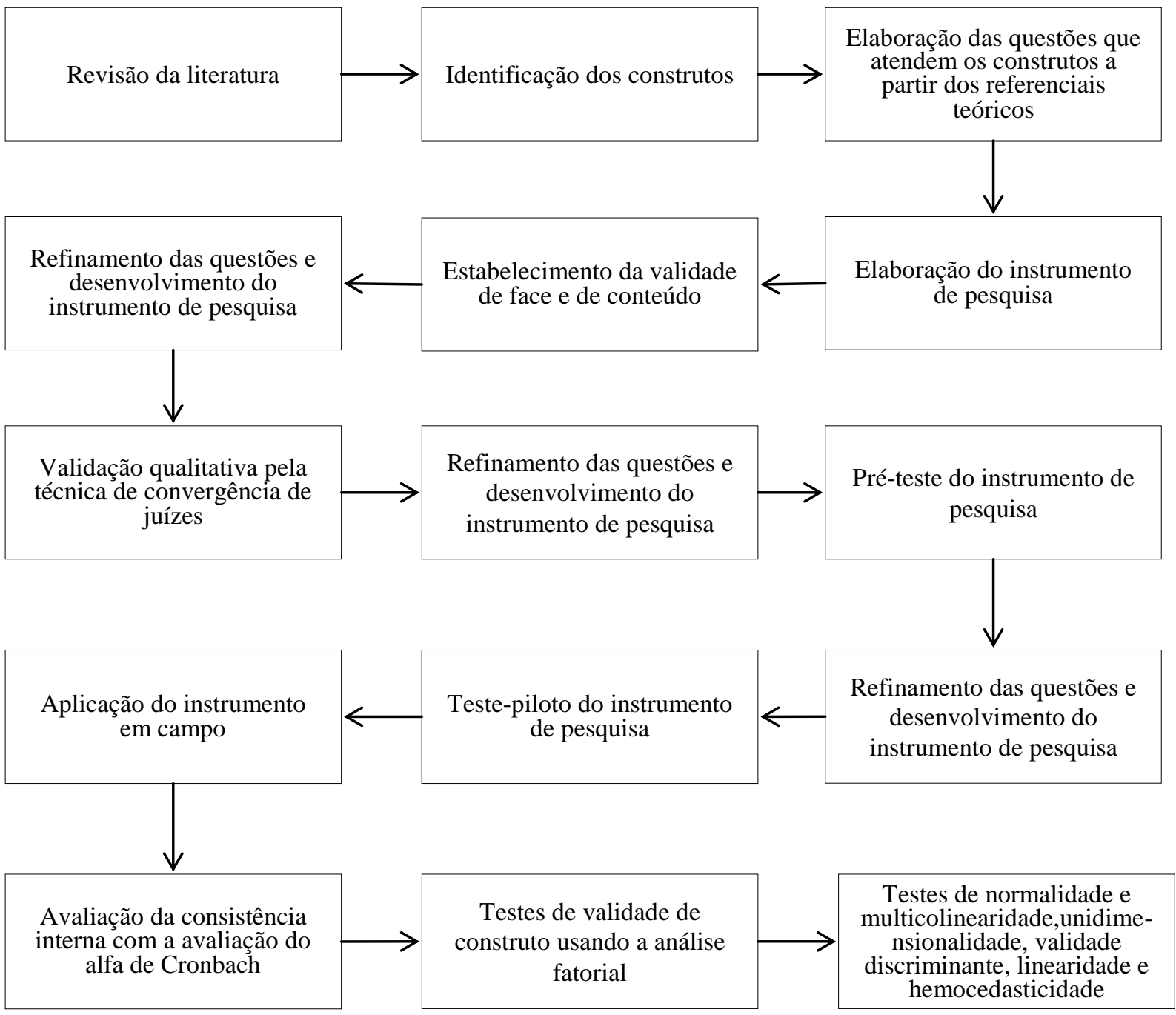

Fonte: Os autores.

\subsection{Validação de face e de conteúdo}

A validação de conteúdo ou de face foi aprofundada através do método de juízes, acadêmicos e práticos (MALHOTRA, 2005). Esse método “[...] visa submeter a escala ao julgamento de experts para que possam julgar a pertinência ou não de cada afirmação para a medição específica que se pretende efetuar" (MATTAR, 1993, p. 34).

A validade de face e de conteúdo foi feita a partir da avaliação por especialistas doutores na área do conhecimento, em cujo foco a presente pesquisa se enquadra. Foram enviados questionários com as variáveis para serem apreciadas por cinco doutores especialistas na área do conhecimento do tema da 
pesquisa e, com isso, foram coletadas informações detalhadas de uma série de melhorias sugeridas. Todas as sugestões de melhoria foram implementadas visando a obtenção da clareza do instrumento de pesquisa e de questões que de fato estivessem alinhadas aos construtos.

Após essa etapa, três empresários com experiência na área de atuação como distribuidor também foram entrevistados, na busca de refinamento e sugestões com aproximação dos termos mais utilizados na linguagem própria das empresas do setor.

\subsection{Validação convergente e confiabilidade pela técnica $Q$-sort}

No estágio seguinte, utilizou-se a técnica Q-sort, seguindo o método de Perreault e Leigh (1989), que atesta a capacidade de relacionar as variáveis/escalas criadas com seus respectivos construtos, visando a obtenção da confiabilidade no tratamento qualitativo das questões que objetivam investigar esses construtos.

O método usado estabelece uma tentativa de propor a medição de forma confiável, na validação interna dos construtos, evitando potenciais problemas de unidimensionalidade deles (STRATMAN; ROTH, 2002). Trata-se do conjunto de variáveis que se relacionam a apenas um construto latente inerente, isto é, variáveis indicadoras que carregam suas cargas sobre apenas um construto e os indicadores têm apenas um conceito em comum (HAIR JÚNIOR et al., 2009). Esse método visa assegurar maior confiabilidade interna do instrumento, antes mesmo de sua aplicação, o que vai evitar problemas posteriores em que a validação da confiabilidade da pesquisa pode ser obtida pela análise do alfa de Cronbach, que, em pesquisas de cunho social, têm sido admitidas como válidas medições acima de 0,70. Essa medida de confiabilidade varia de 0 a 1 , sendo os valores de 0,6 a 0,7 considerados o limite inferior de aceitabilidade (HAIR JÚNIOR et al., 2009).

Esse método solicita a cada juiz fazer uma identificação da questão com o construto tido como o que melhor se relaciona à questão. $\mathrm{O}$ juiz lê a questão e a classifica no construto que considera mais adequado. A avaliação da capacidade de relacionar a variável com o construto confere uma pontuação ou nota. O valor de 1 indica a concordância perfeita do julgamentos entre juízes. Valores de 0,65 ou maiores são considerados aceitáveis como níveis de concordância convergente (STRATMAN; ROTH, 2002). Discordâncias qualitativas entre as avaliações dos juízes podem sinalizar problemas de compreensão da questão, uma vez que o esquema de classificação 
das questões pode ser ambíguo, apesar de as escalas de medição serem nominais. Para tanto, nesse método, o primeiro passo é a estimação da confiabilidade a partir dos julgamentos das questões ligadas aos construtos pelos juízes.

As questões com problemas foram modificadas ou descartadas e uma segunda rodada foi realizada. Os mesmos juízes que participaram da técnica Q-sort na primeira rodada também participaram na segunda rodada. Ao final da segunda etapa, foi possível verificar novamente as questões problemáticas para os devidos ajustes. Esse processo chegou a uma terceira fase, em que uma parcela total dos juízes novamente avaliou as questões modificadas e fez novas classificações no seus respectivos construtos.

\subsection{Juízes participantes da pesquisa}

O instrumento de coleta de dados construído foi enviado, então, para treze doutores da área de administração e engenharia, os quais foram os juízes que avaliaram os itens/escalas do questionário e os classificaram em cada construto apresentado. Todos esses juízes participaram das duas primeiras rodadas de avaliação, e oito deles participaram da terceira etapa.

\subsection{Questões utilizadas em cada construto}

O Quadro 1 apresenta as questões e seus respectivos construtos que foram as apresentadas aos juízes para a participação na técnica Q-sort, representando o primeiro esforço de validação do questionário.

O questionário, dessa forma, foi composto de 20 variáveis, todas elas medidas subjetivas e ligadas aos construtos, como se pode ver pelo Quadro 1. O instrumento finalizado com a ligação a cada construto e com as definições usadas, está apresentado no Apêndice A, que contém o instrumento finalizado e editado na web/internet. 
Quadro 1 - Fundamentação teórica para a elaboração do questionário de pesquisa

\begin{tabular}{|c|c|c|}
\hline Construto & Descrição & Embasamento Teórico \\
\hline $\begin{array}{l}\text { Monitoramento do } \\
\text { Desempenho }\end{array}$ & $\begin{array}{l}\text { Avaliação dos distribuidores sobre o } \\
\text { desempenho }\end{array}$ & $\begin{array}{l}\text { Adaptado de Wagner (2009); Krause et al. } \\
\text { (2006); Krause (2000); Krause et al. (2000); } \\
\text { Wagner (2006); Giunipero (1990); Hahn et } \\
\text { al., (1990); Modi; Malbert (2007). }\end{array}$ \\
\hline $\begin{array}{l}\text { Envolvimento } \\
\text { Direto }\end{array}$ & $\begin{array}{l}\text { Visitas nos distribuidores pelo pessoal } \\
\text { do fabricante para ajudar a programar } \\
\text { melhorias e vice-versa }\end{array}$ & $\begin{array}{l}\text { Adaptado de Krause (2000); Krause et al. } \\
\text { (2000); Wagner (2006); Hartley; Choi } \\
\text { (1996); Newman; Rhee (1990); Hunter et } \\
\text { al. (1996); Langfield-Smith; Greenwood } \\
\text { (1998); Humphreys et al. (2004). }\end{array}$ \\
\hline $\begin{array}{l}\text { Transferência de } \\
\text { Conhecimentos }\end{array}$ & $\begin{array}{l}\text { Capacitação através de treinamentos } \\
\text { técnicos e operacionais e de educação } \\
\text { corporativa }\end{array}$ & $\begin{array}{l}\text { Adaptado de Krause (2000); Monczka et al. } \\
\text { (1993); Galt; Dale (1991); Humphreys et al. } \\
\text { (2004). }\end{array}$ \\
\hline $\begin{array}{l}\text { Desempenho } \\
\text { Operacional }\end{array}$ & Desempenho nos processos operacionais & $\begin{array}{l}\text { Adaptado de Arbache (2004); Wagner } \\
\text { (2006); Krause (2000); Hahn et al. (1990); } \\
\text { Paiva et al. (2008); Spriggs (1994); Kahn } \\
\text { (1998). }\end{array}$ \\
\hline $\begin{array}{l}\text { Desempenho do } \\
\text { Canal }\end{array}$ & $\begin{array}{l}\text { Resultados do negócio/Desempenho } \\
\text { geral do canal de distribuição e vendas }\end{array}$ & $\begin{array}{l}\text { Adaptado de Arbache (2004); Paiva et al. } \\
\text { (2008); Kahn (1998); Jaworski; Kohli } \\
\text { (1993). }\end{array}$ \\
\hline
\end{tabular}

Fonte: elaborado pelo autor.

Para a fase da avaliação das questões de base, que formam diretamente os construtos exógenos, além das traduções das questões, o maior cuidado foi dado na ligação delas com os construtos. Essa ligação diz respeito a potenciais ambiguidades na ligação da questão com o respectivo construto e está diretamente relacionada com a qualidade nominal das questões.

As questões referentes aos construtos "monitoramento do desempenho" e "envolvimento direto" foram avaliadas com a seguinte escala Likert de 5 pontos: 1 - nunca; 2 - até $1 \mathrm{vez}$ no ano; 3 - até 1 vez no semestre; 4 - até 1 vez no bimestre; 5 - até 1 vez ou mais no mês. As questões do construto "transferência de conhecimento" foram avaliadas com a seguinte escala Likert de 5 pontos: 1 - discordo totalmente; 2 discordo; 3 - nem discordo, nem concordo; 4 - concordo; 5 - concordo totalmente. Por fim, as questões referentes aos construtos "desempenho operacional" e "desempenho do canal de distribuição" foram avaliadas com a seguinte escala Likert de 5 pontos: 1 - muito pior; 2 - pior; 3 - igual; 5 - melhor; 6 - muito melhor. 
Quadro 2-Questionário inicial

Monitoramento do desempenho

O fabricante avalia nosso desempenho com um sistema de avaliação formal tendo diretrizes e regras bem estabelecidas.

O fabricante nos dá feedback sobre os resultados do nosso desempenho.

O fabricante usa os resultados da avaliação formal para classificar o desempenho da nossa empresa.

A partir do monitoramento do nosso desempenho, o fabricante define nossas metas de melhorias.

Envolvimento direto

O fabricante tem assistentes técnicos que visitam nossa empresa e se envolvem diretamente conosco.

O fabricante tem gerentes/supervisores que visitam nossa empresa e se envolvem diretamente conosco.

Nosso pessoal faz visitas regulares na fábrica, envolvendo-se diretamente com o pessoal do fabricante.

$\mathrm{O}$ fabricante se envolve diretamente conosco dentro da nossa empresa realizando melhorias nos nossos processos de vendas.

O fabricante se envolve diretamente conosco dentro da nossa empresa ajudando a realizar melhorias na revenda de componentes.

Transferência de conhecimento

O fabricante tem contribuído com treinamentos para o aumento do nosso conhecimento técnico sobre os produtos.

O fabricante tem contribuído com treinamentos para o aumento do nosso conhecimento sobre o mercado.

O fabricante tem contribuído com treinamentos para o aumento do nosso conhecimento sobre os serviços de assistência técnica que prestamos.

Desempenho operacional

Temos tido menos reclamações de clientes.

Os custos operacionais têm se reduzido.

Os prazos de entrega dos nossos serviços têm se reduzido.

Temos conseguido melhorias em todos os nossos processos.

Desempenho do canal de distribuição

Nossa empresa tem obtido retorno superior sobre o capital investido nos últimos 3 anos comparativamente com os concorrentes.

A participação de mercado da nossa empresa comparativamente com os concorrentes é:

A lucratividade da nossa empresa comparativamente com os concorrentes é:

O aumento de vendas de nossa empresa comparativamente com os concorrentes é:

Fonte: os autores.

\subsection{Pré-teste e teste-piloto}

Para obter um instrumento com mais chances de atender os critérios de validade e confiabilidade, o questionário inicial foi sujeito a um pré-teste e a um teste-piloto. O pré-teste foi realizado com respondentes que atuam na área comercial dos canais de distribuição e vendas. Os resultados do pré-teste serviram para realizar pequenas alterações no vocabulário das questões do questionário, sem alterações significativas na sua estrutura.

O teste-piloto foi realizado após as alterações decorrentes do pré-teste e foi aplicado em uma amostra de 15 respondentes que também atuam na área comercial dos canais de distribuição e vendas, mantendo as características da 
população-alvo da pesquisa. Os resultados também foram utilizados para guiar possíveis melhorias nas questões criadas para mensurar os construtos.

\subsection{Análise fatorial confirmatória}

Por fim, o instrumento foi sujeito a uma etapa quantitativa, com o objetivo de permitir a condução de testes de análise fatorial confirmatória. Essa etapa contou com a participação de 199 distribuidores e teve o objetivo de determinar as propriedades psicométricas do instrumento de coleta de dados, confirmando a validade e a confiabilidade do instrumento desenvolvido no estudo. Para essa etapa, foram analisadas as informações de ajuste do modelo e as informações sobre confiabilidade. A maioria das empresas que participaram da pesquisa quantitativa tem mais de 10 anos de atuação como distribuidor (77\%), mais de 50 funcionários $(51 \%)$ e até 50 milhões de reais de faturamento anual $(73 \%)$.

\section{RESULTADOS}

A constatação na primeira avaliação foi que havia muita ambiguidade nas questões relacionadas aos construtos, evidenciando que as perguntas estavam muito genéricas e pouco ligadas ao que elas de fato pretendiam medir. Uma possível explicação para essa constatação está vinculada às questões feitas para representar os construtos do modelo de análise.

As primeiras questões elaboradas não distinguiam de forma precisa tais construtos. A partir dessa percepção, foram refeitas as questões que apresentaram mais divergências na avaliação qualitativa, originando um segundo questionário, que buscou diferençar ao máximo os construtos de forma que o modelo de análise fosse privilegiado, no sentido de buscar uma melhor aproximação com o modelo de análise proposto.

$\mathrm{O}$ índice de concordância foi calculado pelo percentual de respostas nas quais os juízes indicam corretamente a qual construto aquela questão pertence. Portanto, se temos hipoteticamente um grupo de avaliadores de 10 juízes e a questão $n 1$ pertencente a um construto A1 apresenta um índice de convergência de $70 \%$, esse valor indica que 7 juízes consideraram a questão como integrante do construto A1, como esperado. Os demais juízes a consideraram como pertencente a outros construtos An. O grau de convergência de cada construto foi calculado pela média aritmética dos graus de concordância das variáveis que pertencem a esse construto. Nesse caso, a segunda rodada de avaliação mostrou um grau de convergência médio da ordem de $58,8 \%$, como se pode ver pela Tabela 1 . 
Esse resultado reflete o esforço de alteração do questionário e das questões, levando em conta a organização alcançada com a relação entre os construtos. Isto é, foram feitas alterações que estivessem alinhadas e suportadas pelas relações entre os construtos, permitindo que as questões fossem desenvolvidas utilizando-se uma terminologia que deixasse mais claro o significado de cada construto. Entretanto, o valor da convergência deveria ser de pelo menos $0,62(62 \%)$ para que a confiabilidade estimada para a amostra fosse de no mínimo 0,70, valor esse aceitável para os estudos sociais. De acordo com Hair Júnior et al. (2009), nesse nível há uma razoável indicação da adequação da validade discriminante.

Tabela 1 - Resultado da segunda pesquisa qualitativa para avaliar o grau de convergência entre juízes

\begin{tabular}{|c|c|c|c|c|c|}
\hline Construto $^{\dagger}$ & $\begin{array}{c}\text { Construto 1 } \\
\text { Monitoramento } \\
\text { do Desempenho }\end{array}$ & $\begin{array}{c}\text { Construto } 2 \\
\text { Envolvimento } \\
\text { Direto } \\
\end{array}$ & $\begin{array}{c}\text { Construto } 3 \\
\text { Transferência de } \\
\text { Conhecimentos }\end{array}$ & $\begin{array}{l}\text { Construto } 4 \\
\text { Desempenho } \\
\text { Operacional }\end{array}$ & $\begin{array}{c}\text { Construto } 5 \\
\text { Desempenho } \\
\text { do Canal }\end{array}$ \\
\hline $\begin{array}{c}\text { Grau de } \\
\text { Convergência }\end{array}$ & $74 \%$ & $50 \%$ & $60 \%$ & $50 \%$ & $60 \%$ \\
\hline
\end{tabular}

$(\dagger)$ Grau de convergência médio entre todos os construtos: $58,8 \%$.

Fonte: os autores.

Tendo em vista que a confiabilidade projetada seria baixa, fez-se uma terceira modificação nos itens do instrumento com a reformulação das questões, visando melhor capacidade de interpretação pelos respondentes a fim de aumentar o poder da validade convergente, isto é, melhorar a capacidade de validade e da unidimensionalidade.
$\mathrm{Na}$ terceira fase, os dados de validade convergente indicaram um percentual médio de $81 \%$ na concordância entre os juízes, o que aponta uma estimação de confiabilidade na ordem de $85 \%$ para a amostra final (Tabela 2).

Tabela 2 - Resultado da terceira pesquisa qualitativa para avaliar o grau de convergência entre juízes

\begin{tabular}{cccccc}
\hline Construto ${ }^{\dagger}$ & $\begin{array}{c}\text { Construto 1 } \\
\text { Monitoramento } \\
\text { do Desempenho }\end{array}$ & $\begin{array}{c}\text { Construto 2 } \\
\text { Envolvimento } \\
\text { Direto }\end{array}$ & $\begin{array}{c}\text { Construto 3 } \\
\text { Transferência de } \\
\text { Conhecimentos }\end{array}$ & $\begin{array}{c}\text { Construto 4 } \\
\text { Desempenho } \\
\text { Operacional }\end{array}$ & $\begin{array}{c}\text { Construto 5 } \\
\text { Desempenho do } \\
\text { Canal }\end{array}$ \\
\hline $\begin{array}{c}\text { Grau de } \\
\text { Convergência }\end{array}$ & $80 \%$ & $86 \%$ & $76 \%$ & $80 \%$ & $83 \%$ \\
\hline
\end{tabular}

$(\dagger)$ Grau de convergência médio entre todos os construtos: $81 \%$.

Fonte: os autores.

Para melhor qualificar os índices obtidos, a Tabela 3 a seguir mostra os valores de convergência obtidos individualmente para cada questão. Como se pode ver, os construtos identificados possuem altos índices de convergência, 
acima do mínimo necessário preconizado por Perreault e Leigh (1989). O percentual total do bloco do construto foi calculado pela média aritmética dos percentuais de concordância de todas as questões que compõem o construto. Observa-se também que a questão 4 obteve um índice de concordância baixo, questão também alterada e ajustada antes da elaboração do instrumento para o pré-teste.

Tabela 3 - Resultado por questão da terceira pesquisa qualitativa para avaliar o grau de convergência entre juízes

\begin{tabular}{|c|c|c|c|}
\hline & Questão & \% de concordância & $\begin{array}{l}\text { Percentual total do } \\
\text { bloco do construto }\end{array}$ \\
\hline \multirow{4}{*}{$\begin{array}{l}\text { Construto } 1 \text { Monitoramento } \\
\text { do Desempenho }\end{array}$} & 1 & 100 & \multirow{4}{*}{$80 \%$} \\
\hline & 2 & 67 & \\
\hline & 3 & 100 & \\
\hline & 4 & 55 & \\
\hline \multirow{7}{*}{$\begin{array}{c}\text { Construto } 2 \text { Envolvimento } \\
\text { Direto }\end{array}$} & 5 & 77 & \multirow{7}{*}{$86 \%$} \\
\hline & 6 & 89 & \\
\hline & 7 & 89 & \\
\hline & 8 & 89 & \\
\hline & 9 & 89 & \\
\hline & 10 & 89 & \\
\hline & 11 & 77 & \\
\hline \multirow{4}{*}{$\begin{array}{c}\text { Construto } 3 \text { Transferência de } \\
\text { Conhecimentos }\end{array}$} & 12 & 89 & \multirow{4}{*}{$76 \%$} \\
\hline & 13 & 89 & \\
\hline & 14 & 89 & \\
\hline & 15 & 78 & \\
\hline \multirow{4}{*}{$\begin{array}{c}\text { Construto } 4 \text { Desempenho } \\
\text { Operacional }\end{array}$} & 16 & 77 & \multirow{4}{*}{$80 \%$} \\
\hline & 17 & 77 & \\
\hline & 18 & 77 & \\
\hline & 19 & 89 & \\
\hline \multirow{4}{*}{$\begin{array}{c}\text { Construto } 5 \text { Desempenho do } \\
\text { Canal }\end{array}$} & 20 & 77 & \multirow{4}{*}{$83 \%$} \\
\hline & 21 & 89 & \\
\hline & 22 & 89 & \\
\hline & 23 & 77 & \\
\hline
\end{tabular}

Fonte: os autores.

Em todos os testes feitos com os juízes, os itens foram dispostos aleatoriamente com o objetivo de o respondente não identificar o construto pela sua sequência lógica nem por eventual conexão sequenciada, evitando-se com isso uma tendenciosidade nos resultados.

\subsection{Análise fatorial confirmatória}

Foi realizada uma análise fatorial confirmatória em duas etapas, dentro de uma proposta de nested models, ou seja, reunindo os construtos em dois grupos que tivesses maior relação com o objetivo de dar maior robustez a cada modelo testado.

Desse modo, inicialmente foi testado o modelo que compreendeu os construtos monitoramento de desempenho, envolvimento direto e transferência de conhecimentos. Os resultados apresentados ficam dentro do esperado tanto em relação à carga das 
variáveis (todas acima de acima de 0,48) também apresentaram valores acima do 0,7 quanto aos dos índices de robustez do recomendados por Nunnaly (1979), modelo. Os índices Alfa de Cronbach, que variando de 0,80 a 0,90 , apresentados na indicam a confiabilidade da escala, Tabela 4 a seguir.

Tabela 4 - Resultados da Análise Fatorial Confirmatória dos Construtos Básicos $\dagger$

\begin{tabular}{|c|c|}
\hline Medidas* & $\begin{array}{c}\text { Carga } \\
\text { Padronizada }\end{array}$ \\
\hline $\begin{array}{l}\text { Monitoramento do Desempenho } \\
\text { O fabricante avalia nosso desempenho com um sistema de avaliação formal tendo } \\
\text { diretrizes e regras bem estabelecidas. }\end{array}$ & .872 \\
\hline O fabricante nos dá feedback sobre os resultados do nosso desempenho. & .873 \\
\hline $\begin{array}{l}\text { O fabricante usa os resultados da avaliação formal para classificar o desempenho da } \\
\text { nossa empresa. }\end{array}$ & .811 \\
\hline $\begin{array}{l}\text { Envolvimento Direto } \\
\text { O fabricante tem gerentes/supervisores que visitam nossa empresa e se envolvem } \\
\text { diretamente conosco. }\end{array}$ & .777 \\
\hline $\begin{array}{l}\text { Nosso pessoal faz visitas regulares na fábrica, envolvendo-se diretamente com o } \\
\text { pessoal do fabricante. }\end{array}$ & .480 \\
\hline $\begin{array}{l}\text { O fabricante se envolve diretamente conosco dentro da nossa empresa realizando } \\
\text { melhorias nos nossos processos de vendas. }\end{array}$ & .786 \\
\hline \multicolumn{2}{|l|}{ Transferência de Conhecimento } \\
\hline $\begin{array}{l}\text { O fabricante tem contribuído com treinamentos para o aumento do nosso } \\
\text { conhecimento técnico sobre os produtos. }\end{array}$ & .636 \\
\hline $\begin{array}{l}\text { O fabricante tem contribuído com treinamentos para o aumento do nosso } \\
\text { conhecimento sobre os clientes. }\end{array}$ & .903 \\
\hline $\begin{array}{l}\text { O fabricante tem contribuído com treinamentos para o aumento do nosso } \\
\text { conhecimento sobre o mercado. }\end{array}$ & .868 \\
\hline
\end{tabular}

† Índices de ajuste para a Análise Fatorial Confirmatória: $\chi^{2}=33.92$, d.f. $=24, \mathrm{p}<.086, \mathrm{RMR}=0,05$, RMSEA $=0,04, \mathrm{GFI}=, 966, \mathrm{AGFI}=0.936, \mathrm{NFI}=0,963, \mathrm{IFI}=0,989, \mathrm{CFI}=0,989$.

*Alfa de Cronbach: Monitoramento do Desempenho (0,90), Envolvimento Direto $(0,80)$, Transferência de Conhecimento $(0,83)$.

Fonte: os autores.

Complementarmente, foram também testados os dois construtos de desempenho, dentro da proposta de nested models (BURT, 1971; GERBING; ANDERSON, 1985) e da orientação seguida para os demais construtos relativos ao modelo inicial proposto. Para todas as escalas de desempenho, solicitou-se que a análise fosse feita em relação aos últimos três anos. Também se indicava ao respondente que cada empresa fosse comparada aos seus principais competidores, variando em uma escala do tipo Likert de 1 (muito pior) até 5 (muito 
melhor). Novamente, os resultados mostraram-se bastante satisfatórios, tanto em relação às cargas fatoriais obtidas como em relação aos índices de robustez do modelo testado, como mostra a Tabela 5 a seguir. As cargas ficaram acima de 0,50 e os demais índices de robustez ficaram acima dos valores esperados. Os índices relativos ao Alfa de Cronbach também ficaram acima do esperado, sendo igual a 0,73 para desempenho operacional e 0,84 para desempenho do negócio.

Tabela 5 - Resultados da Análise Fatorial Confirmatória dos Construtos de Desempenho ${ }^{\dagger}$

\begin{tabular}{l|c}
\hline \multicolumn{1}{c|}{ Medidas* } & $\begin{array}{c}\text { Carga } \\
\text { Padronizada }\end{array}$ \\
\hline $\begin{array}{l}\text { Desempenho Operacional } \\
\text { Os custos operacionais têm se reduzido. }\end{array}$ & .561 \\
Os prazos de entrega dos nossos serviços têm se reduzido. & .758 \\
Temos conseguido melhorias em todos os nossos processos. & .718 \\
Desempenho do Negócio \\
A participação de mercado da nossa empresa comparativamente com os concorrentes é: \\
A lucratividade da nossa empresa comparativamente com os concorrentes é: & .619 \\
O aumento de vendas de nossa empresa comparativamente com concorrentes é: & .515 \\
\hline
\end{tabular}

$\uparrow$ General statistics for goodness-of-fit for the CFA: $\chi^{2}=33.92$, d.f. $=24, p<.086$, RMR $=0.05$, RMSEA $=$ $0.04, \mathrm{GFI}=.966, \mathrm{AGFI}=0.936, \mathrm{NFI}=0.963, \mathrm{IFI}=0.989, \mathrm{CFI}=0.989$.

*Alfa de Cronbach: Desempenho Operacional $(0,73)$, Desempenho do Negócio $(0,84)$.

Fonte: os autores.

\section{CONCLUSÕES}

A contribuição significativa e objeto do presente estudo diz respeito à utilização de métodos de validação em estudos cujas teorias ainda não foram consolidadas por estudos anteriores, como o método de validação qualitativa convergente de Perreault e Leigh (1989). Com o método utilizado no presente estudo, evita-se que um questionário seja enviado a campo sem maior clareza quanto à sua validade inicial. Em muitos casos, os pesquisadores, ao elaborar um questionário, não têm variáveis claramente definidas, pois não existiram estudos anteriores sobre o tema em estudo. Com isso, há o risco de se ter pesquisas em que as variáveis e os construtos apresentarão problemas por vezes insolúveis na etapa final de validação com técnicas como análise fatorial exploratória ou análise fatorial confirmatória. Dessa forma, o esforço do pesquisador e a qualidade dos achados poderão ficar comprometidos.

Os resultados indicam que a técnica Q-Sort permite uma validade inicial plenamente aceitável para os construtos desempenho do canal, desempenho 
operacional, transferência de conhecimentos, monitoramento do desempenho e pelo envolvimento direto, relacionados ao tema de integração entre fabricante e distribuidor.

Há uma expectativa de que a transferência de conhecimentos ocorra através do envolvimento direto e isso tem impacto direto sobre o desempenho operacional e o desempenho do canal, como já constatado por estudos anteriores sobre estratégias adotadas pelos fabricantes para o desenvolvimento de seus fornecedores.

Da mesma forma como proposto em Dyer e Hatch (2006) na díade fabricante-fornecedor, este estudo sugere que o repasse de conhecimentos como uma rotina de transferência de conhecimentos pode levar a um desempenho superior do canal de distribuição. Possivelmente, essa rotina de troca de conhecimentos eleve também o capital relacional entre os agentes envolvidos, como se viu no embasamento teórico, gerando um relacionamento entre as empresas que dificilmente poderia haver em empresas individualmente.

Dessa modo, um fabricante pode criar e sustentar uma vantagem competitiva através da sua rede de distribuidores, implementando recursos intangíveis de conhecimentos em seus canais. Essa pode ser uma vantagem embasada na integração dos distribuidores com o fabricante via repasse de conhecimentos, que se opera de forma tácita.

No presente estudo, pelo método proposto por Perrealt e Leigh (1989), foi possível avaliar previamente o índice de confiabilidade dos construtos acima de 0,8 . Com isso, houve assertividade na ação prévia à pesquisa de campo, evitando-se a obtenção dessa constatação apenas após a coleta de dados.

Outro aspecto que convém salientar diz respeito à inclusão de variáveis além do mínimo aconselhável para a robustez de uma escala, que usualmente é em torno de três itens. Assim, com a análise na etapa da validação convergente, foi possível a retirada daquelas que apresentaram os menores índices de cargas padronizadas. Se a pesquisa se inicia com o número “mínimo de pelo menos 3 variáveis por constructo" (HAIR JÚNIOR et al., 2009, p. 596) e as cargas padronizadas não suportam o construto proposto, então o tratamento de dados ficará potencialmente prejudicado.

Este estudo indica que quando o pesquisador não tem escalas consolidadas para uma dada pesquisa, em razão de novos temas ou teorias pouco exploradas, pode ser utilizada uma ampliação inicial do número de variáveis nos construtos propostos. O refinamento das variáveis 
ocorrerá a partir das etapas de validação desenvolvidas, reduzindo o construto final àquelas variáveis que de fato têm maior significância estatística.

Como limitação principal do estudo, o questionário foi aplicado em apenas uma indústria, o que indica cautela quanto aos resultados e aos construtos encontrados. As mesmas análises poderão ser feitas em outros setores para verificar se os resultados permanecem próximos aos encontrados.

\section{REFERÊNCIAS}

ARBACHE, F. C. et al. Gestão de Logística, Distribuição e Trade Marketing. Rio de Janeiro: FGV, 2004.

ARGOTE, L.; P. INGRAM. Knowledge transfer: A basis for competitive advantage in firms. Organizational Behavior and Human Decision Processes, v. 82, p. 150169, 2000.

AUGIER, M.; TEECE, D. J. Dynamic capabilities and the role of managers in business strategy and economic performance. Organization Science, v. 20, n. 2, p. 410-421, 2009.

BELLIN, H. Best practice channel management: the channel management framework. Journal of Marketing Channel, v. 14, p. 117-127, 2006.

BOWERSOX, D. J.; CLOSS, D. J.; COOPER, M. B. Gestão logística de cadeias de suprimentos. Porto Alegre: Bookman, 2006.

BURT, R. S. Interpretational confounding of unidimensional variables in structural equations modeling. Sociological Methods and Research, v. 5, p. 3-51, 1971.

DYER, J. H.; HATCH, N. W. Relationspecific capabilities and barriers to knowledge transfer: Creating advantage through network relationships. Strategic Management Journal, Hoboken, NJ, v. 27, p. 701-719, 2006.

DYER, J. H.; SINGH, H. The relational view: cooperative strategy and sources of inter-organizational competitive advantage. Academy of Management Review, v. 23, p. 660-679, 1998.

DYER, J.; NOBEOKA, K. Creating and managing a high performance knowledgesharing network: the Toyota case.

Strategic Management Journal, v. 21, n. 3, p. 345-367, 2000.

FRAZIER, G. L. Organizing and managing channels of distribution. Journal of the Academy of Marketing Science, v. 27, n. 2, p. 226-240, 1999.

FROHLICH, M. T.; WESTBROOK, R. Arcs of Integration: an international study of supply chain strategies. Journal of Operation Management, v. 19, p. 185200, 2001.

GALT, J. D. A.; DALE, B. G. Supplier development: a British case study.

International Journal of Purchasing and Materials Management, v. 27, n. 1, p. 1622, 1991.

GERBING, D. W.; ANDERSON, J. C. The effects of sampling and model characteristics on parameter estimation for Maximum Likelihood Confirmatory Factor Analysis. Multivariate Behavioral Research, v. 20, n. 3, p. 255-271, 1985.

HAIR JÚNIOR, J. F. et al. Análise multivariada de dados. 6 . ed. Porto Alegre: Bookman, 2009. 
HANDFIELD, R. B. et al. Avoid the pitfalls in supplier development. MIT Sloan Management Review, v. 41, n. 2, p. 37-49, 2000.

HARTLEY, J.; CHOI, T. Supplier development: customers as a catalyst of process change. Business Horizons, v. 39, p. 37-44, 1996.

HEIDE, J.; JOHN, G. Do norms matter in marketing relationship. Journal of Marketing, v. 56, p. 32-44, 1992.

HUMPHREYS, P. K.; LI, W. L.; CHAN, L. Y. The impact of supplier development on buyer-supplier performance. Omega, $n$. 32, p. 131-143, 2004.

HUNTER, L.; BEAUMONT, P.; SINCLAIR, D. A partnership route to human resource management. Journal of Management Studies, vol. 33, n. 2, p. 235-257, 1996.

LANGFIELD-SMITH, K.;

GREENWOOD, M. R. Developing cooperative buyer-supplier relationships: a case study of Toyota. Journal of Management Studies, vol. 35, n. 3, p. 331-353, 1998.

JAWORSKI, B. J.; KOHLI, A. K. Market orientation: antecedents and consequences. Journal of Marketing, v. 57, p. 53-70, 1993.

KOGUT, B.; ZANDER, U. Knowledge of the firm, combinative capabilities, and the replication of technology. Organization Science, v. 3, n. 3, p. 383-397, 1992.

KRAUSE, D. R. Supplier development: current practices and outcomes.

International Journal of Purchasing and Materials, v. 33, n. 2, p. 12-19, 1997.

KRAUSE, D. R.; HANDFIELD, R. B.; SCANNELL, T. V. An empirical investigation of supplier development: reactive and strategic processes. Journal of Operations Management, v. 17, n. 1, p. 39-58, 1998.

KRAUSE, D. The antecedents of buying firms' efforts to increase suppliers' performance. Journal of Operations Management, v. 17, n. 2, p. 205-224, 1999.

KRAUSE, D.; SCANNELL, T. V.; CALANTONE, R. J. A Structural analysis of the effectiviness of buying firm's strategies to improve supplier performance. Decision Sciences, v. 31, n. 1, p. 33-55, 2000 .

MALHOTRA, Naresh K. Pesquisa de marketing: uma orientação aplicada. 5. ed. Porto Alegre: Bookman, 2005.

MATTAR, Fauze Najib. Pesquisa de marketing. São Paulo: Atlas, 1993.

MEHTA, R.; DUBINSKY, A. J.; ANDERSON, R. E. Marketing channel management and the sales manager. Industrial Marketing Management, v. 31, p. 429-439, 2002.

MENTZER, J. T. et al. Defining supply chain management. Journal of Business Logistics, v. 22, n. 2, p. 1-25, 2001.

MODI, S. B.; MABERT, V. A. Supplier development: improving supplier performance through knowledge transfer. Journal of Operations Management, Washington, v. 25, n. 1, p. 42-64, 2007.

MONCZKA, R. M.; TRENT, R. J.; CALLAHAN, T. J. Supply base strategies to maximize supplier performance.

International Journal of Physical Distribution and Logistics Management, v. 23, n. 4, p. 42-54, 1993.

NUNNAly, J., Psychometric Theory. New York, McGraw-Hill, 1979.

PERREAULT, W. D. J.; LEIGHT, L. E. Reliability of Nominal Data Based on 
Qualitative Judgments. Journal of Marketing Research, v. 26, p. 135148,1989 .

PORTER, M. E. Competição: estratégias competitivas essenciais. Rio de Janeiro: Campus, 1999.

PRAHINSKI, C.; BENTON, W. C. Supplier evaluations: communication strategies to improve supplier performance. Journal of Operations Management, v. 22, p. 39-62, 2004.

PRAHINSKI, C.; FAN, Y. Supplier evaluations: the role of communication quality. Journal of Supply Chain Management, v. 43, n. 3, p. 16-28, 2007.

PURDY, L.; ASTAD, U.; SAFAYENI, F. Perceived effectiveness of the automotive supplier evaluation process. International Journal of Operation and Production Management, v. 14, n. 6, p. 91-103, 1994.

SHIN, H.; COLLIER, D. A.; WILSON, D. D. Supply management orientation and supplier/buyer performance. Journal of Operations Management, v. 18, n. 3, p. 317-333, 2000.

SPENDER, J. C. Making knowledge the basis of a dynamic theory of the firm.

Strategic Management Journal, v. 17, p. 45-62, 1996.

STRATMAN, J. K.; ROTH, A. V. Enterprise resource planning (ERP) competence constructs: two-stage multiitem scale development and validation.

Decision Sciences, v. 33, n. 4, p. 601-628, 2002.

SZULANSKI, G. Exploring internal stickiness: impediments to the transfer of best practice within the firm. Strategic Management Journal, v. 17, p. 27-43, 1996.

TEECE, D. J. Explicating dynamic capabilities: the nature and microfoundations of (sustainable) enterprise performance. Strategic Management Journal, v. 28, p. 13191350, 2007.

TEECE, D. J.; PISANO, G.; SHUEN, A. Dynamic Capabilities and strategic management. Strategic Management Journal, v. 18, n. 7, p. 509-533, 1997.

WAGNER, S. M. A firm's responses to deficient supplier and competitive advantage. Journal of Business Research, v. 59, n. 6, p. 686-695, 2006.

WAGNER, S. M.; KRAUSE, D. Supplier development: communications approaches activities and goals. International Journal of Production Research, v. 47, n. 12, p. 3161-3177, 2009.

WAHAB, S. A.; ROSE, R. C.; OSMAN, S. I. W. Measuring the effects of technology suppliers' characteristics on degree of inter-firm technology transfer based on knowledge-based view and organizational learning perspective. International Business Research, v. 4, n. 4, p. 53-61, 2011.

WATTS, C. A.; HAHN, C. K. Supplier development programs: an empirical analysis. International Journal of Purchasing and Material Management, v. 29, n. 2, p. 11-17, 1993.

WILLIAMSON, O. The economic institutions of capitalism. New York: Free Press, 1985.

WILSON, D. T. An integrated model of buyer-seller relationships. Journal of the Academy of Marketing Science, v. 23, n. 4, p. 335-345, 1995.

WINTER, S. Four Rs of profitability: Rents, resources, routines, and replication. In: MONTGOMERY, C. A. (Ed.).

Resource-based and evolutionary theories of the firm: towards a synthesis. Boston: Kluwer Academic, 1995. 
WREN, B. M. Channel structure and strategic choice in distribution channels.

Journal of Management Research, v. 7 , n. 2, p. 78-86, Aug. 2007.

ZHOU, H.; BENTON JÚNIOR, W. C. Supply chain practice and information sharing. Journal of Operations Management, v. 25, n. 6, p. 1348-1365, 2007. 